\title{
Oxidant/Anti-Oxidant Status in Rats Exposed to Simulated Weightlessness by Hind-Limb Unloading and Reloading
}

\author{
Parimal Chowdhury* and Michael Soulsby
}

\author{
Department of Physiology and Biophysics, University of Arkansas for Medical Sciences, 4301 W Markham Street, Little \\ Rock, AR 72205, USA
}

\begin{abstract}
Hindlimb suspension or better known as hind-limb unloading (HLU) of rats is a validated model representing weightlessness, simulating microgravity. This is accomplished by removal of weight-bearing loads from hindquarters producing a cephalic fluid shift. The current study examines whether exposure to microgravity by hind-limb unloading will activate oxidant/antioxidant defense system. Tissue levels of malondialdehyde (MDA), total glutathione (GSH+GS-SG) and superoxide dismutase (SOD) were measured in harvested tissues following HLU and reloading. To rule out any variability in normal dietary oxidant intake, all animals received in their diet Purina rat chow containing $9.6 \%$ soy meal $(4.8 \%$ soy protein). Results show that tissues harvested from rats following 2 weeks of unloading had significantly elevated MDA levels ranging from $0.3-0.8 \mu \mathrm{M} / \mathrm{mg}$ protein when compared to paired loaded controls $(0.2-0.4 \mu \mathrm{M} / \mathrm{mg}$ protein, $\mathrm{p}<$ $0.5)$. Tissues from rats reloaded for 2 weeks following unloading decreased MDA levels from their peak values $(p<0.05)$. GSH levels increased up to $350 \mu \mathrm{M}$ depending on tissues as a result of unloading, and two weeks of reloading decreased GSH levels to $250 \mu \mathrm{M}$ from their peak levels. SOD levels increased in all harvested organs in unloaded group ranging from $0.8-4.0 \mu \mathrm{M}$ with continued increase after two weeks of reloading, $\mathrm{p}<0.05)$. Our data indicate that oxidative stress is induced during unloading as evidenced by increased tissue MDA levels. The response involves an increase in GSH and SOD levels, but two weeks of reloading decreased MDA and GSH levels, while further increasing SOD levels, implying the tissue adaptation to induced oxidative stress.
\end{abstract}

\section{INTRODUCTION}

Weightlessness presents significant physiologic challenges to those in space for extended periods of time. $\mathrm{Nu}-$ merous studies show that space flight may increase oxidative stress [1-3]. However, the results from many short and longterm space flights on the oxidative responses provide conflicting results [4-7]. Employing rat hind limb unloading (HLU), a validated NASA animal model of simulated microgravity representing weightlessness [8], we have evaluated the tissue specific oxidative response both under unloading and reloading conditions in rats fed a basal soy protein diet. Malondialdehyde, superoxide dismutase and glutathione levels have been measured in tissues obtained from control and simulated microgravity induced conditions. This model has been tested over the years in organ systems such as bone and skeletal muscle and is now considered adaptable for ground-based simulated microgravity experiments. The selection of the dietary soy in our current study was based on the fact that soy protein is known to have anti-oxidative properties [9] and is commonly used as a dietary source throughout the world [10].

It is well known that rodents exposed to simulated microgravity show a decreased body weight gain. Undernutrition does not appear to be a likely factor in this phenomenon, for unlike humans, rats are able to maintain food

*Address correspondence to this author at the Department of Physiology and Biophysics, University of Arkansas for Medical Sciences, $4301 \mathrm{~W}$ Markham Street, Little Rock, AR 72205, USA; Tel: (501) 686-5443; Fax: (501)686-8167; E-mail: PChowdhury@uams.edu intake and energy balance after the initial few days of space flight or suspension [11-14]. This suggests that the rodent model is useful to study metabolic effects in response to microgravity in the absence of nutritional deficits observed in humans. It has also been shown that oxidative stress occurs in humans and rats after return to $1 \mathrm{~g}$ following space flight [6]. This is presumably due to decreased antioxidant enzyme activity $[6,15-17]$.

Oxidative stress-induced lipid peroxidation damage in muscle tissue following exposure to microgravity has been well documented in the literature. Matsushima et al. [18], Fomina et al. [19], and Selsby, et al. [17] have each investigated the effects of hind limb-unloading, reloading and immobilization on oxidative damage in skeletal muscle. Onishi et al. [20] reported oxidative stress associated with hind limb unloading and spaceflight resulting in accumulation of mono-ubiquinated LDH in gastrocnemius skeletal muscle suggesting that oxidative stress induces mono- and polyubiquination of LDH-A, which may be involved in lysosomal degradation during HLU. Nikawa et al. [21] investigated key genes for elucidating the mechanisms of skeletal muscle wasting in space. They identified two spaceflight-specific gene expression patterns: (1) an imbalanced expression of genes in the mitochondrial electron transport system, and (2) an up-regulated expression of ubiquitin ligase oxidative stress-induced genes. Their data suggest that oxidative stress may play an important role in triggering protein-ubiquitination in skeletal muscles atrophied by weightlessness.

Vaziri et al. [22] and Sangha, et al. [23] have provided evidence that cardiovascular adaptation to microgravity may 
involve up-regulation of the nitric oxide (NO) vasodilator system. Since it also inhibits renal tubular sodium reabsorption, it may have a major role in the regulation of systemic vascular resistance and blood pressure [38].

An improvement of the imbalance in the pro-oxidant/ antioxidant defense system may lessen the severity of the oxidative stress documented during space flight. Oxidative stress induces an increase in oxidation of membrane fatty acid moieties leading to production of unstable lipid peroxides. These peroxides tend to degrade rapidly into products such as malondialdehyde (MDA) [24]. The formation of these aldehydes contributes significantly to the mechanisms of oxidant-induced injury [25].

We have earlier demonstrated that rats on HLU maintained on a normal Purina diet with $4.5 \%$ soy protein, displayed elevated stress as evidenced by elevated malondialdehyde (MDA) levels in brain tissues harvested after two weeks of suspension [15]. In addition, brain tissues from HLU rats on standard Purina chow showed significantly higher stress, as evidenced by higher MDA levels, than brain tissue from control hind limb-loaded rats maintained on a $37 \%$ soy-protein diet $[9,15]$, suggesting that soy protein in the diet acts as an antioxidant. The current study is designed to test whether this is also true in other tissues harvested. Flavonoids are also known to possess significant antioxidative activity in lipid-aqueous systems [10, 26, 27], and dietary antioxidants may lessen the severity of space flight documented oxidative stress.

In this investigation our first objective was to determine oxidative changes in organs following two weeks of simulated weightlessness (HLU) using Purina rat chow with $9.6 \%$ soy meal or $4.8 \%$ soy protein, and to further evaluate oxidative changes following two weeks of reloading. A basal diet containing higher than the normal soy protein composition in Purina chow was chosen to rule out any variability in normal dietary oxidant intake, and to assure the response is due to HLU under steady state. Our second objective was to determine simultaneously the antioxidant status in these tissues since a large body of literature supports the notion that dietary antioxidants play a role in preventing many human diseases [1, 10, 22, 28-31]. This effect is thought to be due to their ability to increase tissue glutathione (GSH) levels [10, 18] and superoxide dismutase levels which catalyze the dismuation of superoxide into oxygen and hydrogen peroxide [16]. Superoxide dismutase provides an important antioxidant defense in nearly all cells exposed to oxygen [16] by protecting the cell from superoxide toxicity.

\section{MATERIALS AND METHODOLOGY}

Eighteen male Sprague Dawley rats were acclimatized to the Purina rat chow diet with $9.6 \%$ soy meal $(4.8 \%$ soy protein) for 7 days prior to hind limb unloading. Six of the acclimatized rats were non-suspended and maintained on the soy diet for an additional two weeks. Six rats were suspended after acclimatization and maintained on the soy diet for a period of two weeks. A third group of the acclimatized rats was maintained on the soy diet and then suspended for a period of two weeks, followed by hind-limb reloading for an equal period of two weeks. Immediately following each experiment, MDA, total GSH (GSH and GS-SG), and superoxide dismutase (SOD) levels were measured in harvested tis- sues. Total GSH levels were measured because they would be expected to vary with a cell's degree of induction of the GSH antioxidant system. The Cayman GSH Assay Kit uses glutathione reductase for quantification of GSH. The GSH is easily oxidized to the disulfide dimmer GSSG. Hence, both GSH and GSSG are measured, and the Assay reflects total glutathione. SOD levels were measured to determine whether its activation is triggered in concert with lipid peroxidation, for it is known that SOD out-competes the damaging reactions of superoxide, thus protecting the cell from superoxide toxicity [16].

Control values for body weight and food intake and water intake, were recorded for seven days before HLU of the animals at a $30^{\circ}$ angle with the cage floor. Body weights from all animals were monitored on the first day of arrival and again on day seven (the first day of the suspension). Subsequently, the body weights, food, and fluid intakes were monitored on a daily basis until termination of the experiment. The animals were housed in circadian chambers and maintained on 12:12 light-dark cycles. They were allowed free access to tap water and diets. Six non-suspended control rats in group one were maintained on the soy diet for 21 days. Animals in group two were maintained on the soy diet for 21 days, but suspended for only the latter 14 days. Animals in group three were maintained on the soy diet for 35 days, but suspended for 14 days, followed by hind-limb reloading for an equal period of two weeks. The protocol and procedures were in accordance with the Guiding Principles in the Care and Use of Animals of the Council of the American Physiological Society and were approved by the University of Arkansas for Medical Sciences (UAMS) Institutional Animal Care and Use Committee.

No surgical interventions were involved prior to sacrifice. HLU was achieved using a modification of the Morey [8] tail-suspension model. Rats assigned to the unloaded groups were housed individually in Plexiglas chambers $(10 \mathrm{x}$ $19.5 \times 21$ inches). HLU was accomplished with a tail harness constructed by looping a 0.5 x 10-inch Skin-Trac (Zimmer, Inc., Charlotte, NC) orthopedic foam strip around a pulley that can travel along a bar that traverses the length of the cage. The adhesive surfaces along the remainder of the SkinTrac strip were applied to the long axis of opposite sides of the tail, creating a tail-sandwich. This sandwich was encircled by a bias-cut orthopedic stockinette and secured with one-inch glass zip-reinforced strapping tape at the base and tip of the tail. This construction did not interfere with the animal's ability to use its tail to maintain its core body temperature.

Twenty-four hours before sacrifice, the animals were deprived of food but not water. At sacrifice, rats were anesthetized with ketamine hydrochloride and euthanized by decapitation and exsanguination. Liver, pancreas, kidney, small intestine, heart, lung, and brain were harvested.

\section{MDA Assay}

The MDA assay was conducted by the method reported by Esterbauer et al. [24]. Harvested tissues were washed in iced cold $\mathrm{NaCl}$ solution $(9 \mathrm{gm} / \mathrm{L})$. After excising 0.4 to 0.5 grams from each tissue, each sample was homogenized in 20 $\mathrm{mM}$ phosphate buffer, $\mathrm{pH}=7.4$ [tissue to buffer ratio, $1: 10$ w/v]. $10 \mu \mathrm{L}$ of $0.5 \mathrm{M}$ butylated hydroxytoluene per $\mathrm{ml}$ of 
homogenate had been added to prevent additional sample oxidation. The homogenate was centrifuged at 4,000 $\mathrm{g}$ at $4{ }^{\circ} \mathrm{C}$ for 10 minutes. $200 \mu \mathrm{L}$ of supernatant from each homogenate was used to analyze the malondialdehyde (MDA) level in tissue samples. Measurement of MDA is based on the reaction of a chromogenic reagent, N-methyl-2-phenylindole [R1] with MDA at $24{ }^{\circ} \mathrm{C}$. One molecule of MDA interacts with 2 molecules of R1 to yield a stable chromophore with maximal absorbance at $586 \mathrm{~nm}$, and is stable for up to one hour at room temperature. The net absorbance at $586 \mathrm{~nm}$ yields a linear function of MDA ranging from 0 to $20 \mu \mathrm{M}$. The detection limit is $0.1 \mu \mathrm{M}$ of MDA. The final MDA content was expressed as $\mu \mathrm{M}$ of MDA per mg. protein.

\section{Glutathione Assay}

The glutathione assay was conducted following reports published earlier by Baker et al. [32]. Concentrations of oxidized glutathione (GS-SG) in samples were analyzed using a Glutathione Assay Kit from Cayman Chemical Co, Ann Arbor, Michigan (catalogue No. 703002). The kit uses an enzymatic recycling method using glutathione reductase to quantify glutathione (GSH). The GSH produced from the interaction of the sulfhydryl group of GSH with 5, 5' dithiobis-2-nitrobenzoic acid (DTNB) was oxidized to the disulfide dimer GS-SG and reduced to GSH by glutathione reductase. The net absorbance at $405 \mathrm{~nm}$ yields a linear function of GS-SG ranging from 0 to $20 \mu \mathrm{M}$, and these values were extrapolated based on the standard curve provided with the assay kit.

\section{Superoxide Dismutase (SOD) Assay}

Superoxide dismutase was assayed by the method reported by Maier and Chan [33]. The SOD concentration was analyzed using a Superoxide Dismutase Assay Kit from
Cayman Chemical Company, Ann Arbor, MI (catalogue no. 706002). The superoxide radical agents formed by the interaction of xanthine oxidase with hypoxanthine were detected by tetrazolium. One unit of SOD detected is equivalent to the amount of enzyme needed to exhibit $50 \%$ dismutase of superoxide radical. The net absorbance at $450 \mathrm{~nm}$ yields a linear function of SOD ranging from 0 to $30 \mathrm{U} / \mathrm{ml}$. The detection of SOD is $0.1 \mathrm{U} / \mathrm{ml}$, and these values were extrapolated based on the standard curve provided with the assay kit.

\section{Protein Assay}

Protein concentrations were measured using a method described by Bradford [34].

\section{Statistics}

Results were calculated as means \pm standard error of mean (SEM). Statistical significance was determined by One-way ANOVA. A value of $\mathrm{p}<0.05$ was considered significant.

\section{RESULTS}

Body weight, food and water intakes are presented in Table 1. Body weights ( $g$ ) of non-suspended rats increased significantly in three weeks on the soy diet. However, the body weights of the HLU group did not change significantly during the period of suspension but remained significantly lower from the unloaded control, $p<0.05$. Body weights of suspended plus reloaded rats, increased significantly at the end of the reloaded period, however, the their body weight gain remained significantly lower when compared to body weights of suspended and loaded control groups (Table 1).

Food intake (g/day) was significantly decreased in suspended rats when compared to loaded control. Upon reloading the food intake was increased but is not significantly

Table 1. Effects of Hind Limb Unloading and Reloading on Body Weight, Food and Water Intake (Mean \pm SEM)

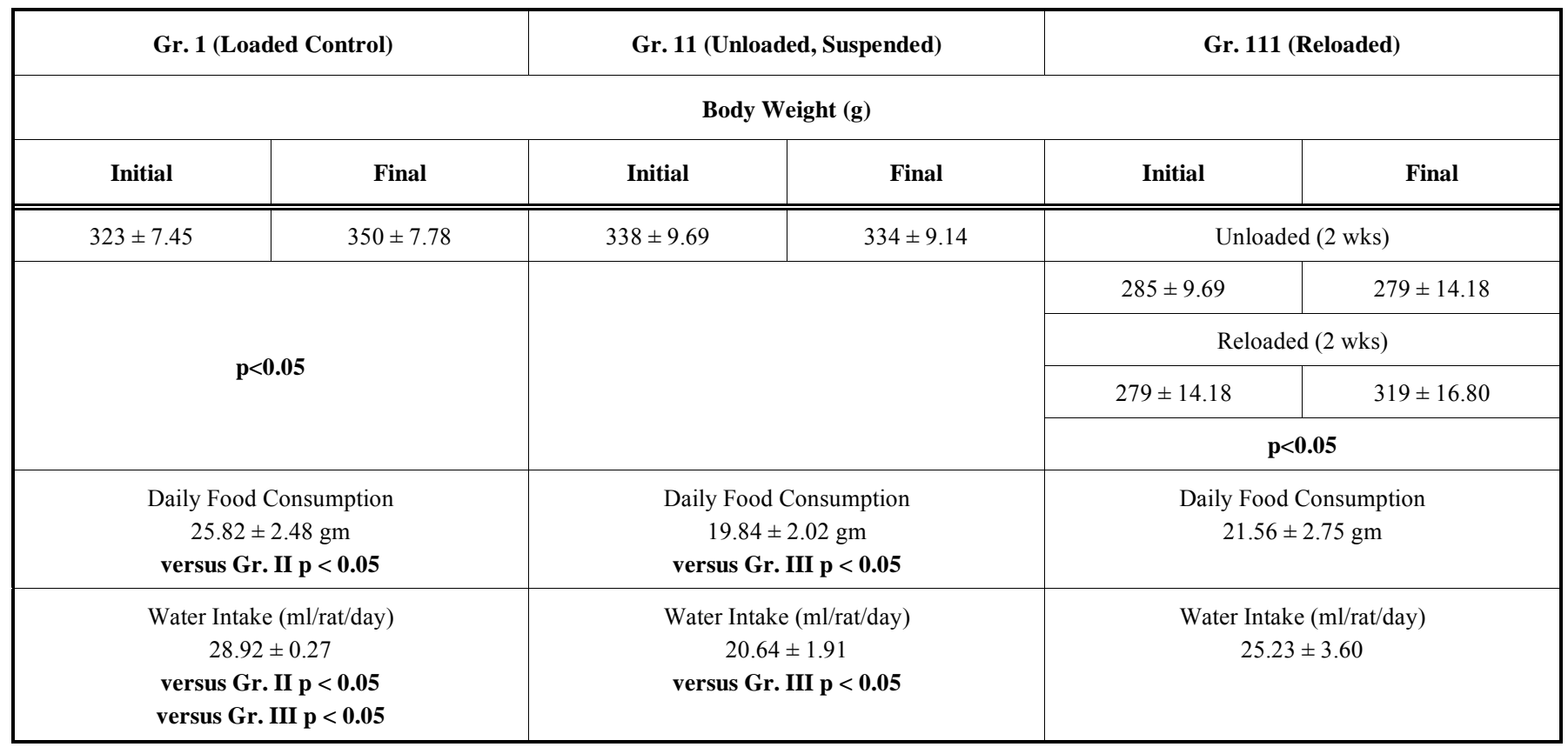

$\mathrm{p}<0.05$, significant differences between the initial and final values. 
different from the suspended group.

Water intake ( $\mathrm{ml} /$ day) was greatest in the loaded control group. Water intake was significantly decreased in the suspended group, and further significantly increased in the reloaded group as compared to unloaded group.

The tissue MDA ( $\mathrm{m} \pm \mathrm{SEM}$ ) levels are shown in Figs. (1 and 2). Fig. (1) represents MDA levels for liver, lung, pancreas and small intestine, while Fig. (2) represents MDA levels for brain, heart and kidney. As shown in Fig. (1), MDA levels in liver, lung, pancreas and small intestine, increased significantly following unloading and decreased upon reloading more significantly in pancreas and small intestine, $(\mathrm{p}<0.05)$.

MDA levels in brain, heart and kidney tissues are shown in Fig. (2). There were significant increases in MDA levels in brain and heart tissues in the unloaded group as compared to the loaded control, $\mathrm{p}<0.05$. These levels decreased toward the basal level upon reloading. In contrast, the MDA levels in the kidney tissues, although increased upon loading as compared to the unloaded control, were not significantly different from either unloaded or reloaded group.

Since dietary antioxidants like soy protein play a role in preventing many human diseases associated with tissue membrane peroxidation by their ability to increase glutathione (GSH) levels in tissues, we measured glutathione levels in harvested tissues. The GSH results are shown in Fig. (3) through 6. Fig. (3) shows that GSH levels for brain and pancreas increased significantly as a result of HLU, and

LMER

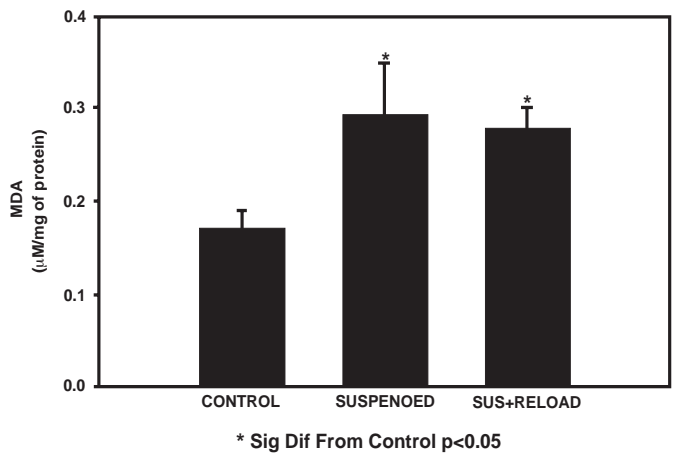

PANCREAS

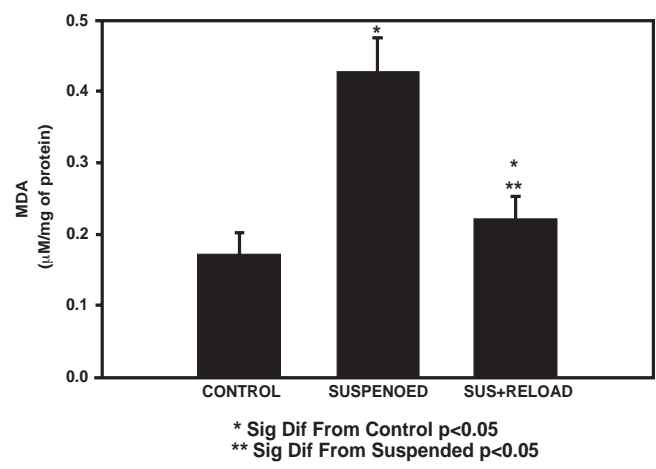

two weeks of reloading decreased GSH levels from their peak levels. The data for GSH levels matched the MDA response in these tissues as shown earlier.

Fig. (4) shows that plasma and lung responded to unloading with an increase in GSH levels, while two subsequent weeks of reloading resulted in a further increase in GSH levels (similar to changes in SOD). Fig. (5) shows GSH levels for liver and heart. GSH levels in these tissues responded to unloading with a decrease in GSH levels, while two weeks of reloading resulted in a further decrease in GSH levels from the unloaded levels. Fig. (6) shows GSH levels in the small intestine which responded to unloading with a decrease in GSH levels which remained significantly below control levels after reloading.

Since intracellular levels of superoxide dismutase (SOD) are increased by exposure to oxygen conferring a greater resistance toward oxygen toxicity, we monitored SOD levels in harvested tissues since SOD might cooperate with GSH in helping to remove free radicals in vivo. Results are provided in Figs. (7 and $\mathbf{8})$.

The results show that all of harvested organs responded to unloading with increased SOD levels, and two weeks of reloading further increased tissue SOD levels $(\mathrm{m} \pm \mathrm{SEM})$.

\section{DISCUSSION AND CONCLUSION}

This study utilizes NASA's hind-limb unloaded (HLU) rat model of exposure to microgravity to monitor cellular

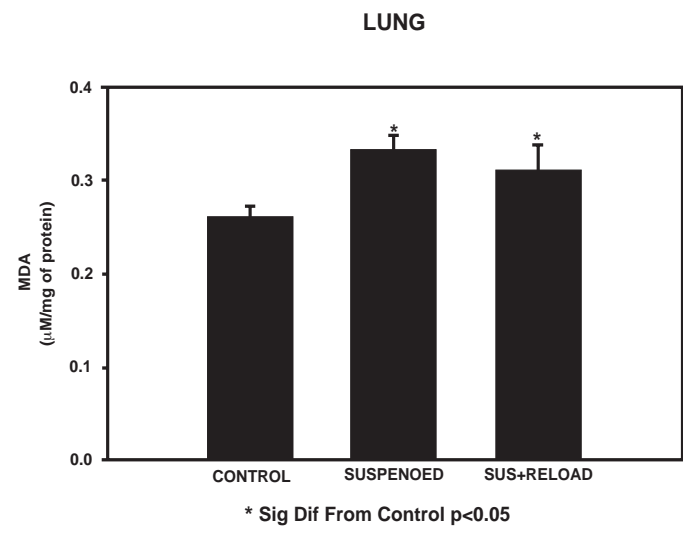

SMALL INTESTINE

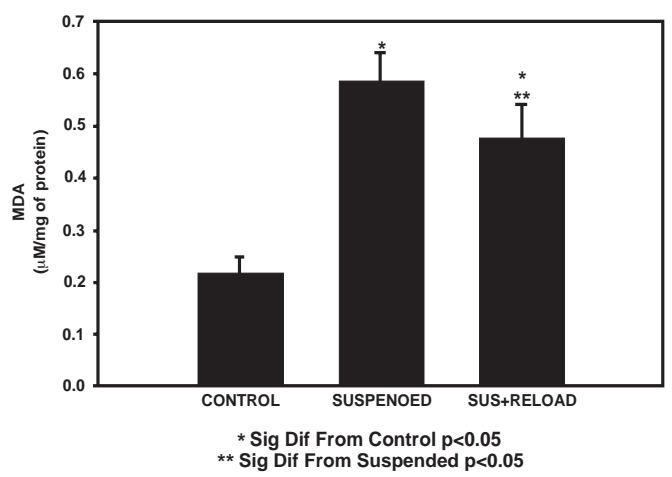

Fig. (1). MDA levels for control, suspended, and reloaded liver, lung, pancreas and small intestine. *, p <0.05 significant differences from control; **, p <0.05 significant differences from the suspended. 
BRAIN

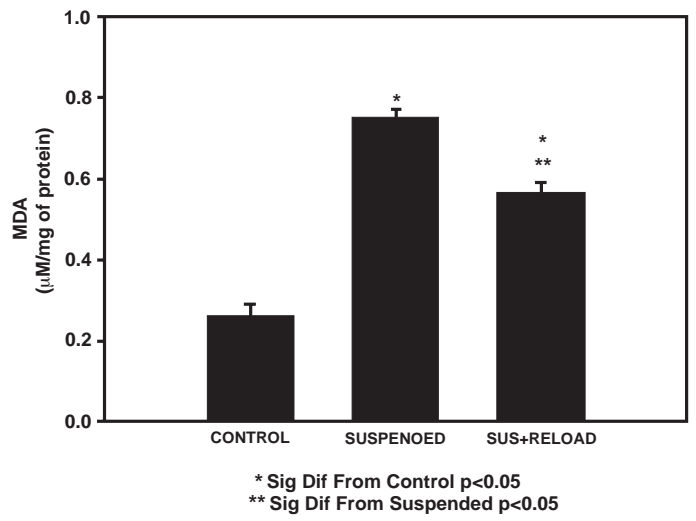

HEART

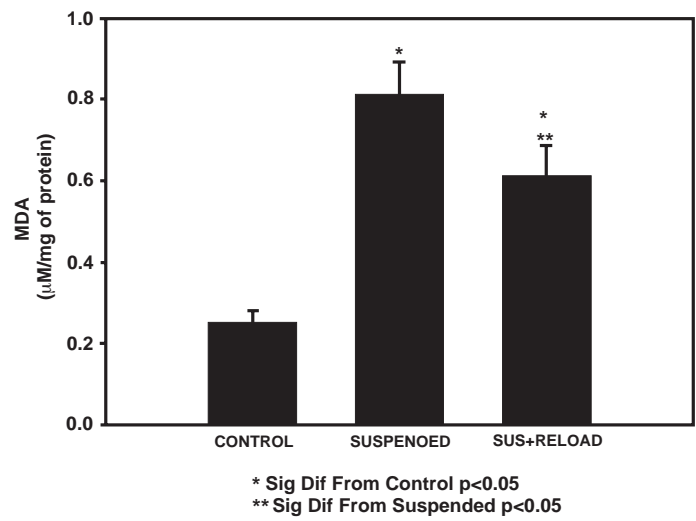

KIDNEY

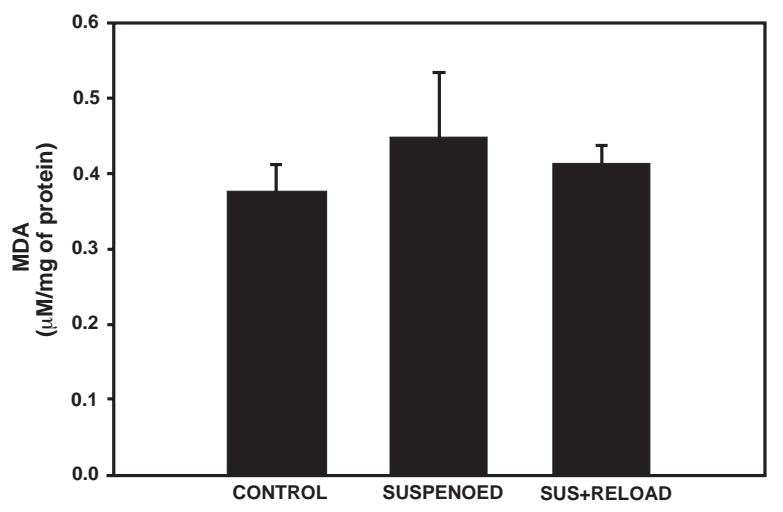

Fig. (2). MDA levels for control, suspended, and reloaded ( $\mathrm{m} \pm \mathrm{SEM}$ ) brain, heart and kidney. *, $\mathrm{p}<0.05$ significant differences from control; **, $\mathrm{p}<0.05$ significant differences from the suspended.

BRAIN

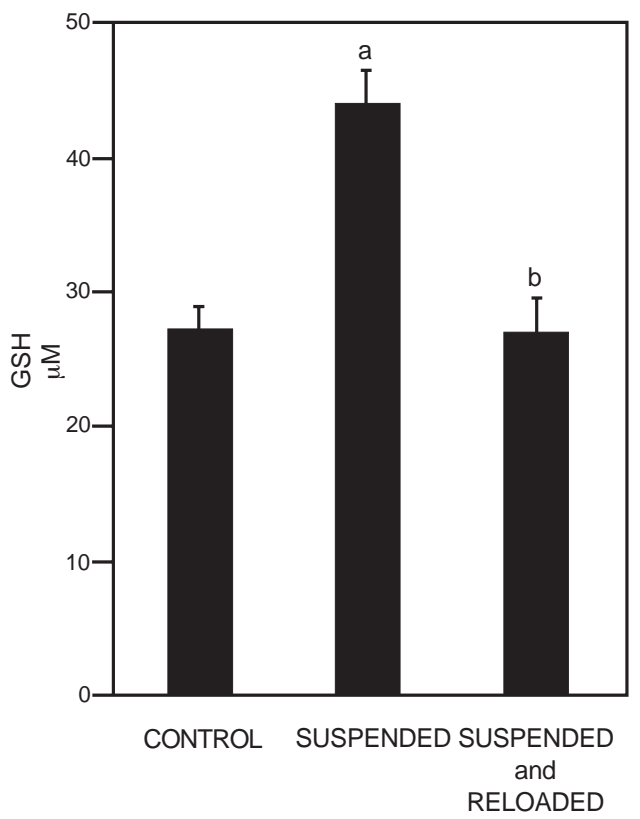

PANCREAS

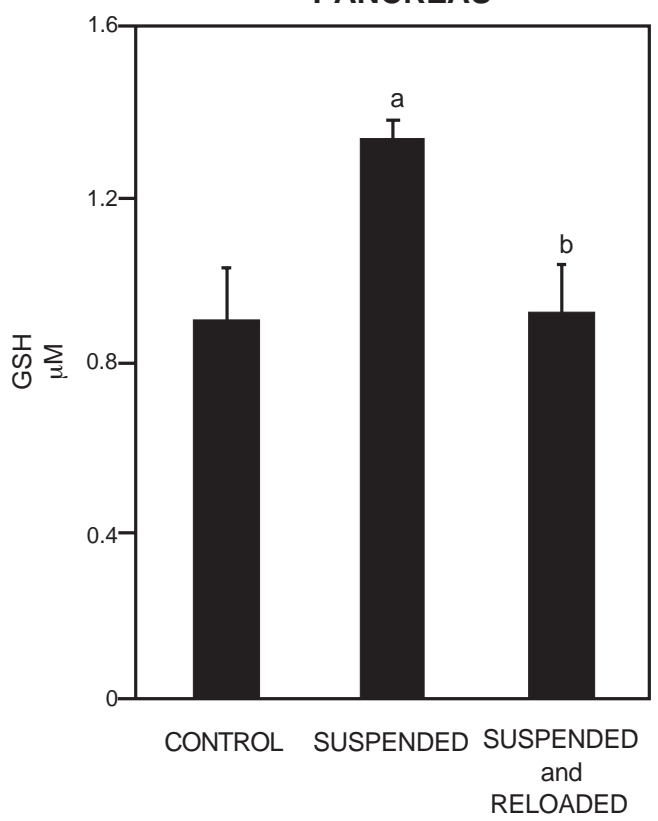

Fig. (3). GSH levels in brain and pancreas $(m \pm S E M) . \mathbf{a}=p<0.05$ significant differences from control; $\mathbf{b}=\mathrm{p}<0.05$ significant differences from the suspended. 

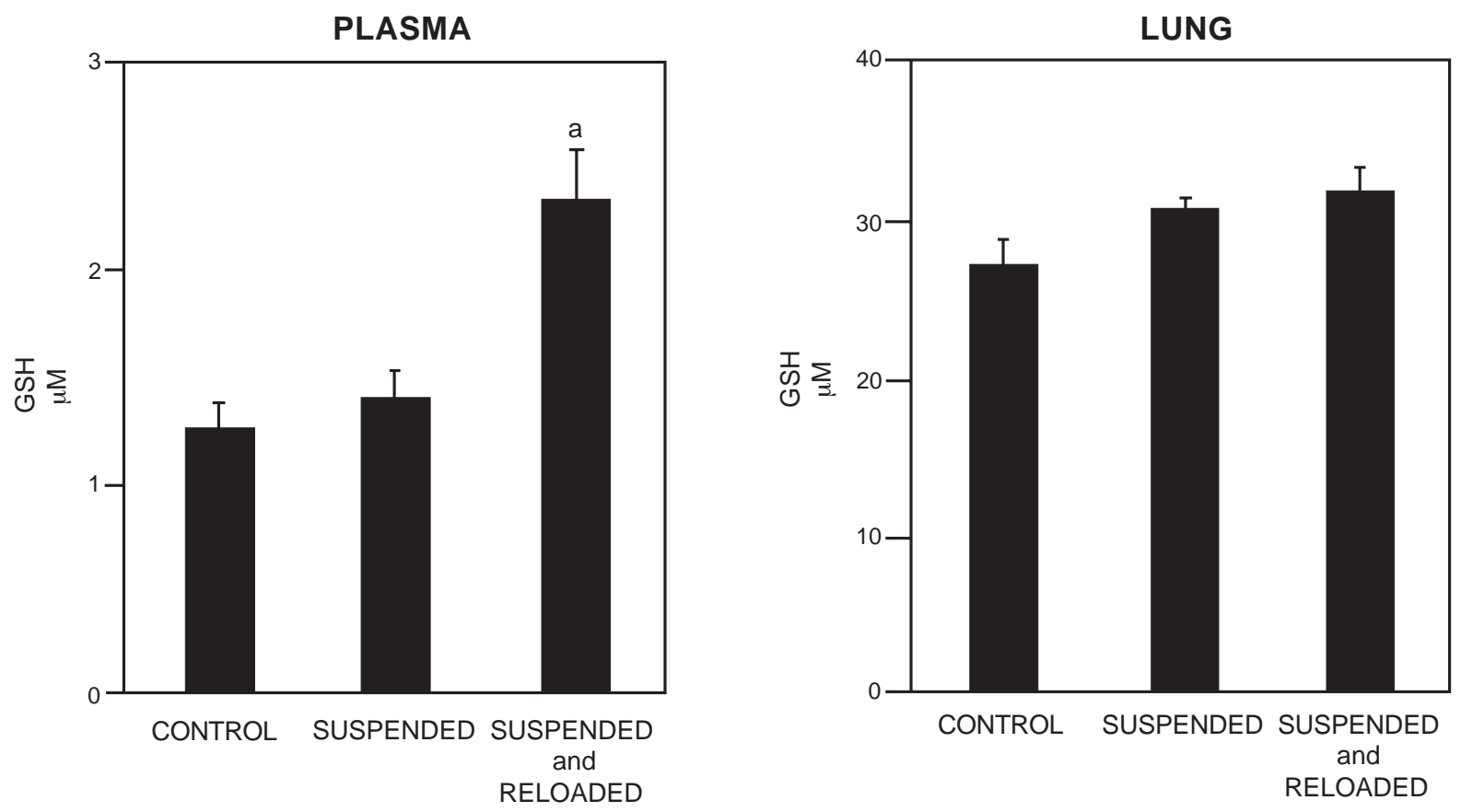

Fig. (4). GSH levels in plasma and lung tissues, $(m \pm$ SEM. $\mathbf{a}=\mathrm{p}<0.05$ significant differences from control).
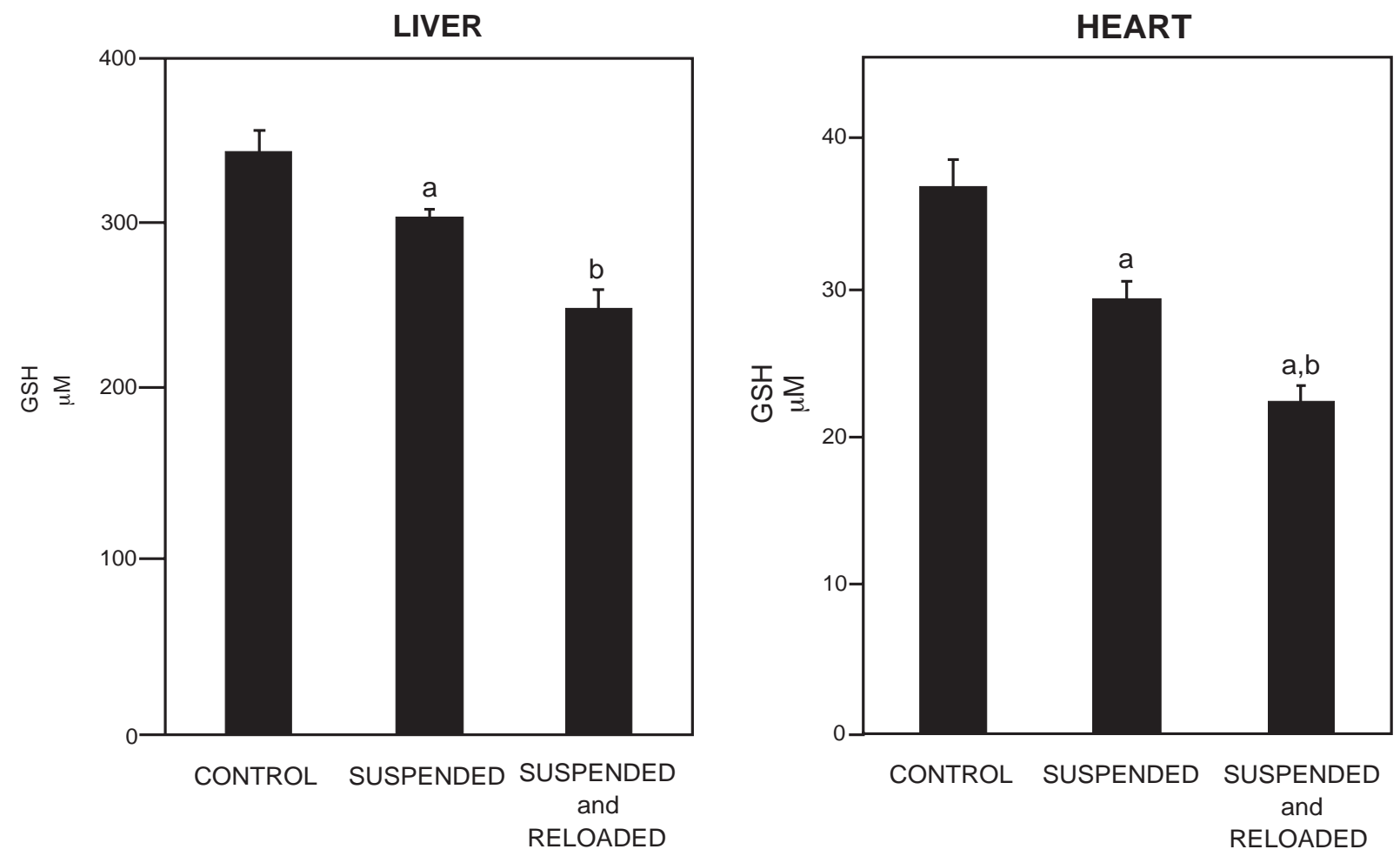

Fig. (5). GSH levels in liver and heart tissues $(\mathrm{m} \pm \mathrm{SEM}) . \mathbf{a}=\mathrm{p}<0.05$ significant differences from control, $\mathbf{b}=\mathrm{p}<0.05$ significant differences from the suspended.

oxidative stress in harvested tissues. Tissue malondialdehyde (MDA) levels are characteristically used to monitor cellular stress as indicators of oxidation of membrane fatty acids. We measured MDA levels in tissues acclimatized to a Purina rat chow diet with $9.6 \%$ soy meal (4.8\% soy protein) for 7 days prior to suspension. The soy protein chow was used to measure the effectiveness of dietary soybean flavonoids as coun- termeasures for the oxidant-antioxidant defense system. Dietary flavonoids found in soy exhibit potent antioxidant activities $[10,26]$ in lipid-aqueous systems. This effect is thought to be due to their ability to increase glutathione (GSH) levels in tissues [10]. Total GSH levels (GSH and GS-SG) were measured in the harvested tissues. 


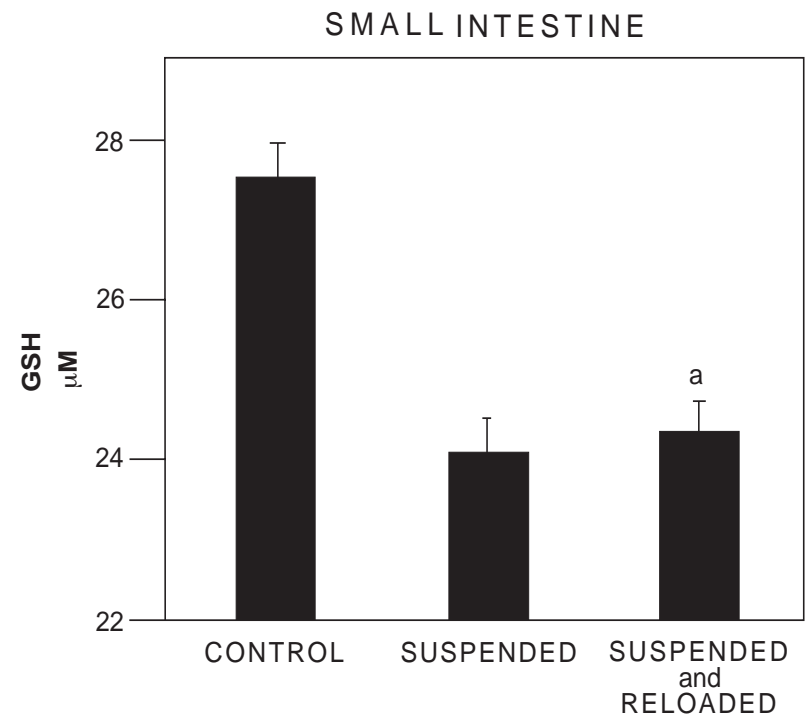

Fig. (6). GSH levels in small intestine ( $m \pm$ SEM). $\mathbf{a}=\mathrm{p}<0.05$ significant differences from control.

HEART

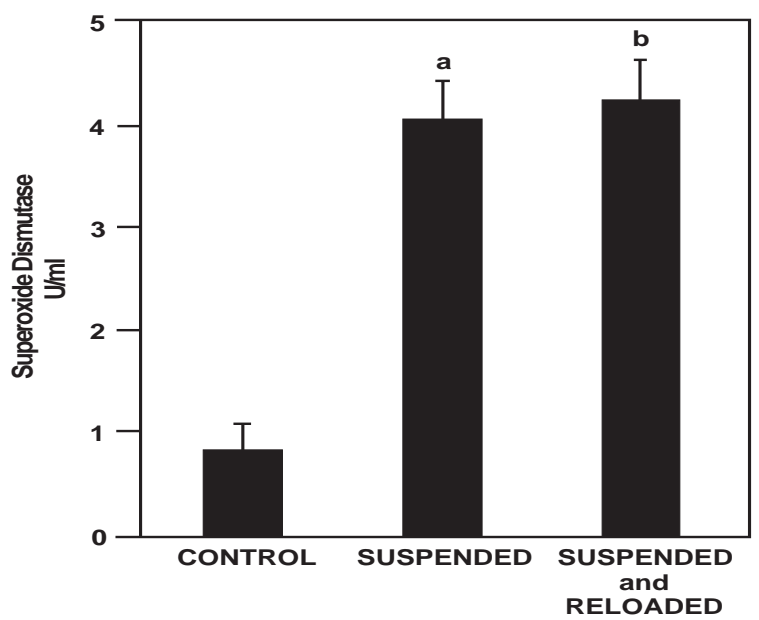

KIDNEY

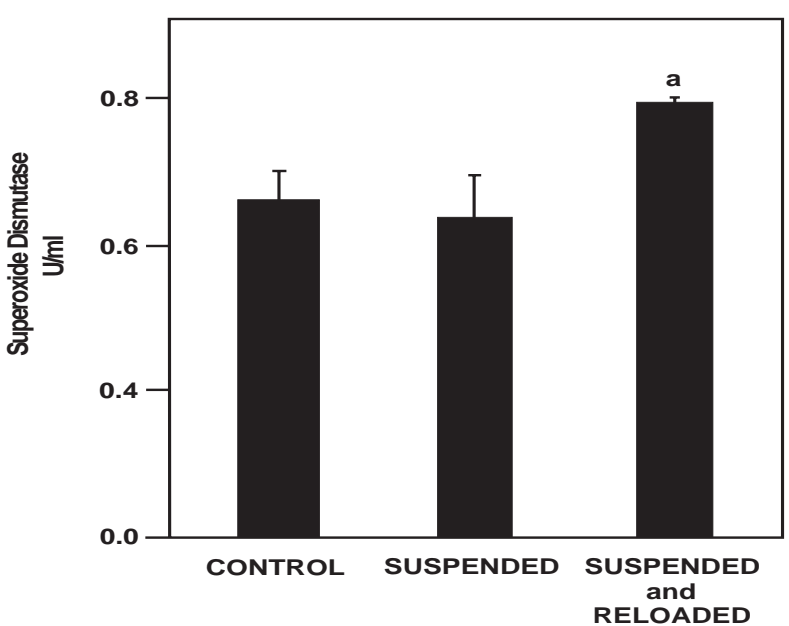

Superoxide dismutase (SOD) levels were also measured in harvested tissues because its toxic product, $\mathrm{H}_{2} \mathrm{O}_{2}$, is removed by glutathione peroxidases utilizing the reducing power of GSH [16]. The product of SOD is $\mathrm{H}_{2} \mathrm{O}_{2}$. Two molecules of GSH are oxidized to form the disulfide-bonded compound, GS-SG in the reduction of a molecule of hydrogen peroxide.

GSH might cooperate with SOD in helping to remove free radicals in vivo. Superoxide is one of the main reactive oxygen species in the cell and, as such, serves role as a key antioxidant. The mutations of this enzyme may cause various pathologies. SOD's generally have high antioxidant capacity [16].

One major function of oxygen metabolism is its interaction with polyunsaturated fatty acids in the process described as lipid peroxidation. When lipid peroxidation occurs in biological membranes there may be gross disturbances in structure/function changes leading to injury or possibly death of the affected cells [35].

Studies conducted on astronauts and cosmonauts to examine tissue lipid oxidative damage have produced conflict-
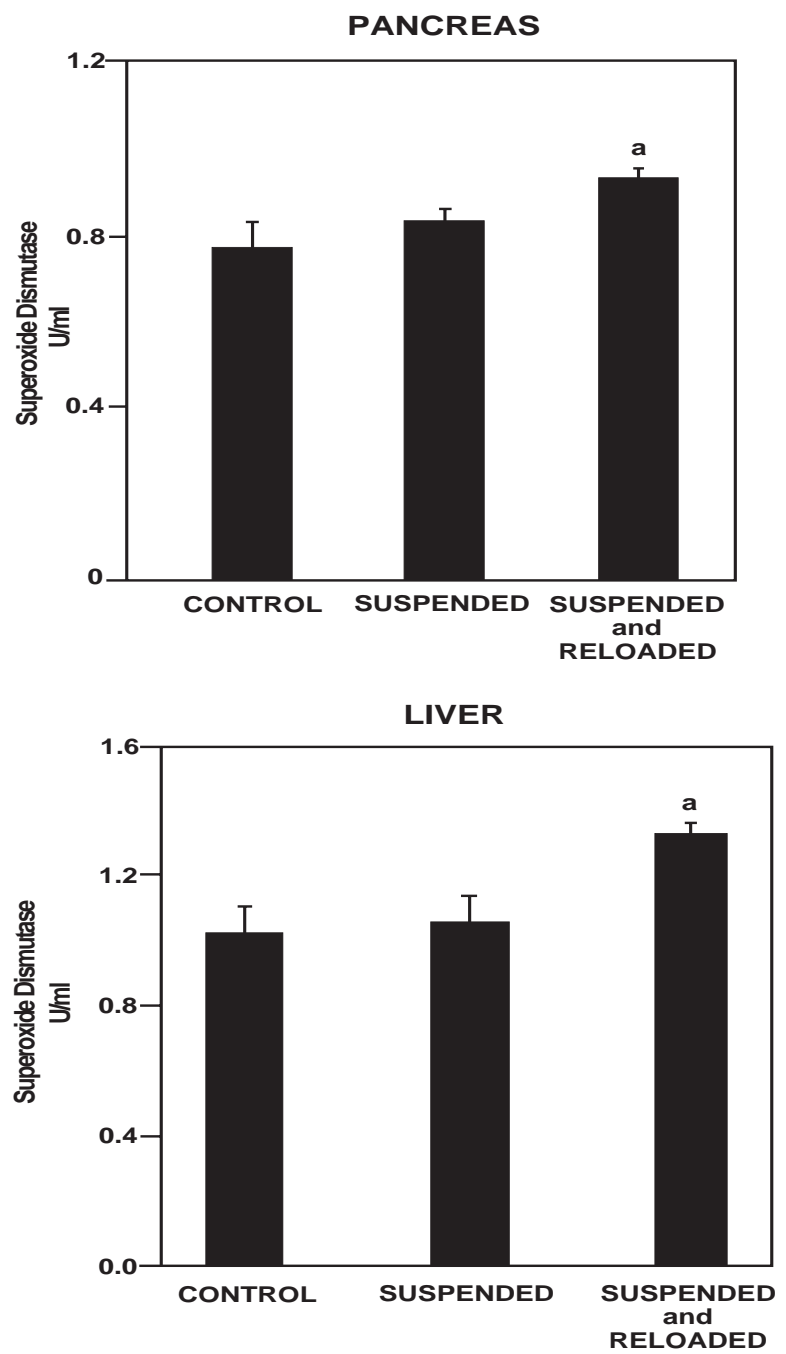

Fig. (7). SOD levels for heart, pancreas, kidney and liver, $(\mathrm{m} \pm \mathrm{SEM}) . \mathbf{a}=\mathrm{p}<0.05$ significant differences from control, $\mathbf{b}=\mathrm{p}<0.05$ significant differences from the suspended. 

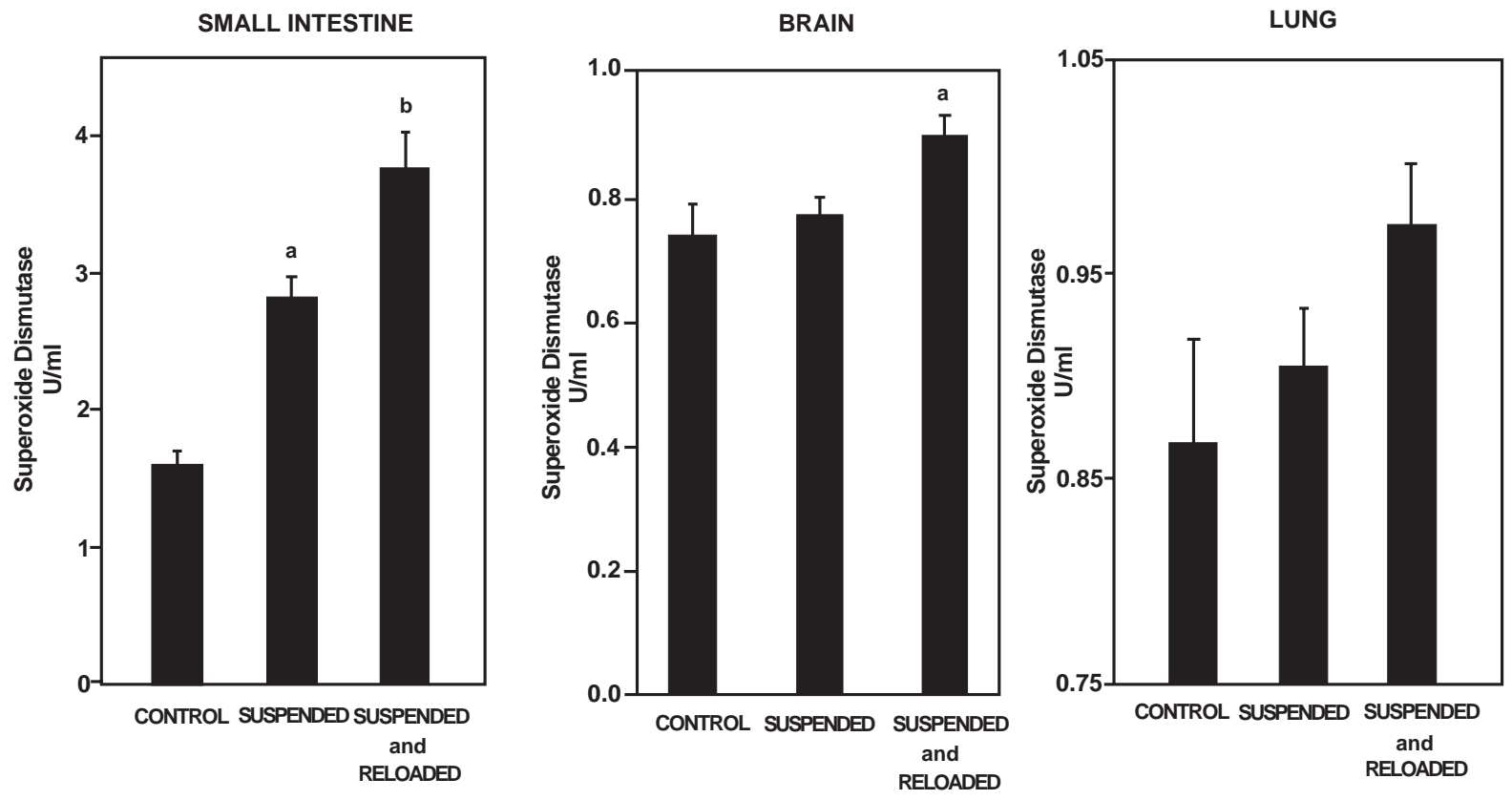

Fig. (8). SOD levels for small intestine, brain and lung, $(\mathrm{m} \pm \mathrm{SEM}) . \mathbf{a}=\mathrm{p}<0.05$ significant differences from control, $\mathbf{b}=\mathrm{p}<0.05$ significant differences from the suspended.

ing results $[4-7,36]$. Observations in rodents returning from space flight $[1,2,37]$ have also produced conflicting results. Thus it is important to reexamine the oxidant/antioxidant status under stress in these animals exposed to simulated microgravity.

It has been proposed that some of the oxidative stress measured in humans and rats is associated with return to $1 \mathrm{~g}$ after space flight [6]. This is presumably due to decreased antioxidant enzyme activity [6, 17]. We demonstrated [15] that hind-limb-unloaded (HLU) rats maintained on a normal Purina diet, displayed elevated malondialdehyde (MDA) levels in tissues harvested immediately after two weeks of suspension. MDA levels were monitored because membrane oxidation produces unstable lipid peroxides which degrade rapidly into malondialdehyde (MDA) [24]. These results confirmed the oxidative stress during HLU, and offered a measure of the degree of stress induced by HLU. However, they did not address tissue oxidation following return to $1 \mathrm{~g}$.

An improvement in the imbalance in the oxidant-antioxidant defense system may lessen the severity of the oxidative stress that has been documented during space flight. A large body of literature supports the notion that dietary antioxidants play a role in preventing many human diseases [1, 2830] associated with lipid peroxidation. Tissue MDA results demonstrate that all organs harvested responded to unloading with a significant increase in MDA levels, except for the kidney. The increase in kidney MDA levels following unloading was not significant. There was no significant difference between MDA levels of non-suspended, suspended only, and reloaded groups in the kidney. These results in the kidney could be related to renal blood flow. Renal blood flow, with the lowest arterio-venous oxygen difference, has ninety percent of its flow diverted to the glomeruli to cleanse the blood, to regulate arterial blood pressure, and to maintain blood oxygen carrying capacity. Only ten percent of the kid- ney's blood flow is used to supply the oxidant-antioxidant defense system. This may partially explain the non-significant differences in MDA levels among the groups studied.

The measured MDA levels in brain, heart, small intestine and pancreas show that 2 weeks of reloading, after 2 weeks of unloading, decreased $(\mathrm{p}<0.05)$ levels from their highest levels, but the values remained significantly higher $(\mathrm{p}<0.05)$ than those for the non-suspended control group. The MDA levels in liver and lung showed that two weeks of reloading, after 2 weeks of unloading, did not significantly decrease ( $>0.05)$ MDA levels from their highest levels, but the values remained significantly higher $(p<0.05)$ than those for the non-suspended control group.

All forms of life maintain a reducing environment within their cells [35]. The best cellular antioxidants are the enzymes superoxide dismutase (SOD) and glutathione peroxidase. GSH peroxidase, together with the hydrogen peroxide generated by SOD, oxidizes glutathione (GSH) to the disulfide dimer GS-SG, which is then reduced to GSH by glutathione reductase. Therefore, SOD might cooperate with GSH in helping to remove free radicals in vivo, so both activities were monitored in tissues in this study.

GSH activities for brain and pancreas were similar to the MDA levels in these tissues. GSH levels increased significantly as a result of HLU, and two weeks of reloading decreased GSH levels from their high after unloading, but the values remained higher that those for the non-unloaded control group. This similarity between MDA and GSH levels could imply that endogenous GSH levels in these tissues were adequate to handle the excess oxidation mirrored by the increased MDA levels.

GSH levels in plasma and lung responded to unloading with an increase, while two weeks of reloading resulted in a further increase in GSH levels. The increase in GSH levels 
followed the increase in SOD levels with unloading in these tissues. GSH levels in liver and heart responded to unloading with a decrease in GSH levels, while two weeks of reloading resulted in a further decrease in GSH levels. GSH levels in the small intestine responded to unloading with a decrease in GSH levels which remained significantly below control levels after two weeks of reloading. If GSH levels were at, or near, their maximums during control studies, then an additional antioxidant system must have been called upon when these organs were exposed to additional stress, as indicated by the MDA levels upon unloading and reloading. As hypothesized earlier, SOD might cooperate with GSH in helping to remove free radicals in vivo, and might be the additional antioxidant system mentioned above.

The SOD activities monitored in this study show that heart, pancreas, kidney, small intestine, brain, lung and liver, which represent all of the organs harvested, responded to unloading with increased SOD levels, and after two weeks of reloading they responded with a further increase in tissue levels.

In conclusion, we have used NASA's hind-limb suspended rat model of exposure to microgravity to monitor total tissue oxidative stress as a function of MDA levels in harvested organ homogenates. This was accomplished in control loaded animals, in animals unloaded for two weeks, and in animals reloaded following two weeks of unloading. Results imply that oxidative stress increases with unloading of an animal, and it decreases toward control levels after two weeks of reloading following unloading. Future studies will be designed to investigate whether a longer period of reloading will reduce the tissue MDA levels back to control levels.

\section{ACKNOWLEDGEMENTS}

This study was supported by a grant from Arkansas Space Grant Consortium. The authors thank Mr. Danny McKay for illustration.

\section{REFERENCES}

[1] Hollander, J.; Gore, M.; Fiebig, R.; Mazzeo, R.; Ohisi, S.; Ohno, H.; Li, L.L. Spaceflight downregulates anti-oxidant defense systems in rat liver. Free Radic. Biol. Med., 1998, 24 (2), 385-90.

[2] Lee, M.D.; Tuttle, R.; Girten, B. Effect of spaceflight on oxidative and anti-oxidant enzyme activity in rat diaphragm and intercostal muscles. J. Gravit. Physiol., 1995, 2(1), P68-69.

[3] Markin, A.A.; Zhuravleva, O.A. Lipid peroxidation and antioxidant defense system in rats after a 14-day space flight in the "Space2044" spacecraft. Aviakosm Ekolog. Med., 1993, 27(1), 47-50.

[4] Stein, T.P. The relationship between dietary intake, exercise, energy balance and the spacecraft environment. Pflugers Arch., 2000, 441 (2-3 Suppl), R21-31.

[5] Stein, T.P. Space flight and oxidative stress. Nutrition, 2002, 18(10), 867-871.

[6] Stein, T.P.; Leskiw, M.J. Oxidant damage during and after spaceflight. Am. J. Physiol. Endocrinol. Metab., 2000, 278 (3), E375-382.

[7] Stein, T.P.; Leskiw, M.J.; Schluter, M.D.; Donaldson, M.R.; Larina, I. Protein kinetics during and after long-term space flight on MIR. Am. J. Physiol., 1999, 276 (6 Pt 1), E1014-21.

[8] Morey-Holton, E.R.; Globus, R.K. Hindlimb unloading of growing rats: A model for predicting skeletal changes during space flight. Bone, 22 (5 Suppl), 83S-88S.

[9] Soulsby, M.E.; Phillips, B.; Chowdhury, P. Brief Communication: The Effects of Soy-protein diet on elevated brain lipid peroxide levels induced by simulated weightlessness. Ann. Clin. Lab. Sci., 2004, 34(1), 103-106.
[10] Ruiz-Larrea, M.B.; Mohan, A.R.; Paganga, G.; Miller, N.J.; Bolwell, G.P.; Rice-Evans, C.A. Antioxidant activity of phytoestrogenic isoflavones. Free Radic. Res., 1997, 26(1), 63-70.

[11] Chowdhury, P.; Soulsby, M.E.; Pasley, J.N. Distribution of ${ }^{3} \mathrm{H}-$ nicotine in rat tissues under the influence of simulated microgravity. Biomed. Environ. Sci., 1999, 12(2), 103-109.

[12] Lane, H.W. Energy requirements for space flight. J. Nutr., 1992, 122(1), 13-18.

[13] Rambaut, P.C.; Leach, C.S.; Leonard, J.I. Observations in energy balance in man during spaceflight. Am. J. Physiol., 1977, 233(5), R208-R212.

[14] Wade, C.E.; Miller, M.M.; Baer, L.A.; Moran, M.M.; Steele, M.K.; Stein, T.P. Body mass, energy intake and water consumption of rats and humans during space flight. Nutrition, 2002, 18(10), 829-36.

[15] Chowdhury, P.; Soulsby, M.E. Lipid Peroxidation in rat brain is Increased by simulated weightlessness and decreased by a soy protein diet. Ann. Clin. Lab. Sci., 2002, 32(2), 188-192.

[16] Fang, Y.Z.; Sun, C.P.; Tian, X.H.; Cong, J.H. Effect of Lu-duo-wei on scavenging superoxide and hydroxyl radicals in vitro. Am. $J$. Chin. Med., 1998, 26(2), 153-8.

[17] Selsby, J.T.; Rother, S.; Tsuda, S.; Pracash, O.; Quindry, J.; Dodd, S.L. Intermittant hyperthermia enhances skeletal muscle regrowth and attenuates oxidative damage following reloading. J. Appl. Physiol., 2007, 102(4), 1702-7.

[18] Matsushima, Y.; Nanri, H.; Nara, S.; Okufuji, T.; Ohta, M.; Hachisuka, K.; Ikeda, M. Hindlimb unloading decrases thioredoxinrelated antioxidant proteins and increases thioredoxin-binding protein-2 in rat skeletal muscle. Free Radic. Res., 2006, 40(7), 715722.

[19] Fomina, E.V.; Davydov, V.V. Age-related characteristics of changes in aldehyde reductase activity in the skeletal muscle of rats during immobilization stress. Biomed. Khim., 2006, 52(4), 378-83.

[20] Onishi, Y.; Hirasaka, K.; Ishihara, I.; Oarada, M.; Goto, J.; Ogawa, T.; Suzue, N.; Nakano, S.; Furochi, H.; Ishidoh, K.; Kishi, K.; Nikawa, T. Identification of mono-ubiquinated LDH-A in skeletal muscle cells exposed to oxidative stress. Biochem. Biophys. Res. Com., 2005, 336(3), 799-806.

[21] Nikawa, T.; Ishihoh, K.; Hirasaka, K.; Ishihara, I.; Ikemoto, M.; Kano, M.; Kominami, E.; Nonaka, I.; Ogawa, T.; Adams, G.R.; Baldwin, K.M.; Yasui, N,; Kishi, K.; Takeda, S. Skeletal muscle gene expression in space-flown rats. FASEB J., 2004, 18(3), 522-4.

[22] Vaziri, N.D.; Ding, Y.; Sangha, D.S.; Purdy, R.E. Upregulation of NOS by simulated microgravity, potential cause of orthostatic intolerance. J. Appl. Physiol., 2000, 89(1), 338-44.

[23] Sangha, D.S.; Vaziri, N.D.; Ding, Y.; Purdy, R.E. Vascular hyporesponsiveness in simulated microgravity: role of nitric oxidedependent mechanisms. J. Appl. Physiol., 2000, 88(2), 507-17.

[24] Esterbauer, H; Schaur, R.J.; Zollner, H. Chemistry and biochemistry of 4-hyroxynonenal, malondaldehyde and related aldehydes: Free Radic. Biol. Med., 1991, 1(1)1, 81-128.

[25] Spitz, D.R.; Elwell, J.H.; Sun, Y.; Oberly, L.W.; Oberly, T.D.; Sullivan, S.J.; Roberts, R.J. Oxygen toxicity in control and $\mathrm{H}_{2} \mathrm{O}_{2}-$ resistant Chinese hamster fibroblast cell lines. Arch. Biochem. Biophys., 1990, 279(2), 249-260.

[26] Record, I.R.; Broadbent, J.L.; King, R.A.; Dreosti, I.E.; Head, R.J.; Tonkin, A.L. Genistein inhibits growth of B16 melanoma cells in vivo and in vitro and promotes differentiation in vitro. Int. $J$. Cancer, 1997, 72(5), 860-64.

[27] Schimchowitsch, S.; Cassel, J.C. Polyamine and aminoguanidine treatments to promote structural and functional recovery in the adult mammalian brain after injury: a brief literature review and preliminary data about their combined administration. J. Physiol. Paris, 2006, 99(2-3), 221-31.

[28] Freedman, B.I.; Wuerth, J.P.; Cartwright, K.; Bain, R.P.; Dippe, S.; Hershon, K.; Mooradian, A.D.; Spinowitz, B.S. Design and baseline characteristics for the aminoguanidine clinical trial in overt type 2 diabetic nephropathy (Action II). Control. Clin. Trials, 1999, 20(5), 493-510.

[29] Jackson, M.J. An overview of methods for assessment of free radical activity in biology. Proc. Nutr. Soc., 1999, 58(4), 1001-6.

[30] Jedidi, I.; Therond, P.; Zarev. S.; Vosson.,C.; Couturier, M.; Massot, C.; Jore, D.; Gardes- Albert, M.; Legrand, A.; BonnefontRousselot, D. Paradoxical protective effect of aminoguanidine toward low-density lipoprotein oxidation : inhibition of apolipoprotein B fragmentation without preventing its carbonylation. Mecha- 
nism of action of aminoguanidine. Biochemistry, 2003, 42(38), 11356-11365.

[31] Yildiz, O.; Ozata, M.; Ozkardes, A.; Deniz, G.; Yildirimkaya, M.; Corakci, A.; Yardim, M.; Gundogan, M.A. Comparison of the effects of aminoguanidine and L-carnitine treatments on somatosensorial evoked potentials in allozan-diabetic rats. Naunyn Schmiedebergs Arch. Pharmco., 1996, 354(4), 526-531.

[32] Baker, M.A.; Cerniglia, G.J.; Zaman, A. Microtiter plate assay for the measurement of glutathione and glutathione disulfide in large numbers of biological samples. Anal. Biochem., 1990, 190(2),360365 .

[33] Maier, C.M.; Chan, P.H. Role of superoxide dismutases in oxidative damage and neurodegeneraive disorders. Neuroscientist, 2002, $8(4), 323-34$.
[34] Bradford, M.M. A rapid and sensitive method for the quantitation of microgram quantities of protein utlizing the principle of proteindye binding. Anal. Biochem., 1976, 72, 248-254.

[35] Davies, K.J.A. Oxidative stress, antioxidant defenses, and damage removal, repair, and replacement systems. IUBMB Life, 2000, 50, 279-289.

[36] Stein, T.P.; Leskiw, M.J.; Schluter, M.D.; Hoyt, R.W.; Lane, H.W.; Gretebeck, R.E.; LeBlanc, A.D. Am. J. Physiol., 1999, 276 (6 Pt 2), R1739-48.

[37] Markin, A.A.; Zhuravleve, O.A.; Balashov, O.I. Lipid peroxidation and activity of diagnostically important blood enzymes in rats during 35 days head-down suspension. Aviakosm Ekolog. Med., 2000, 34 (4), 31-37.

[38] Wu, G.; Menninger, C.J. Regulation of nitric oxide synthesis by dietary factors. Ann. Rev. Nutr., 2002, 22, 61-86.

(c) Chowdhury and Soulsby; Licensee Bentham Open.

This is an open access article licensed under the terms of the Creative Commons Attribution Non-Commercial License (http://creativecommons.org/licenses/ by-nc/3.0/) which permits unrestricted, non-commercial use, distribution and reproduction in any medium, provided the work is properly cited. 